\title{
Cytomegalovirus Glycoprotein B Genotype Distribution in Italian Transplant Patients
}

\author{
Marco Ciottia Eleonora Cellab Massimo Rittàc Massimo Ciccozzi ${ }^{b}$ \\ Rossana Cavallo ${ }^{c}$ Carlo Federico Perno ${ }^{a, d}$ Cristina Costa ${ }^{c}$

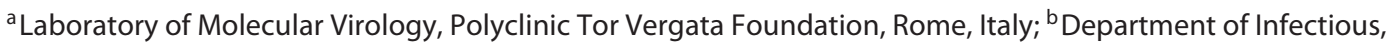 \\ Parasitic and Immunomediated Diseases, National Institute of Health, Rome, Italy; ${ }^{c}$ Microbiology and Virology Unit, \\ University Hospital Città della Salute e della Scienza di Torino, Turin, Italy; ${ }^{\mathrm{d}}$ Department of Experimental Medicine \\ and Surgery, University of Rome Tor Vergata, Rome, Italy
}

\section{Keywords}

Cytomegalovirus · Transplantation · Genetic variation

\section{Abstract}

Background: The cytomegalovirus (CMV) UL55 gene encodes for a glycoprotein implicated in virus pathogenesis. Based on UL55 polymorphism, CMV has been divided into 4 genotypes. Previous studies investigated the possible role of genotypes in the clinical outcome of infection in different categories of patients; however, few data are available, particularly in the transplant setting and Italian case records. Methods: Phylogenetic analysis through a maximum likelihood tree was used to evaluate the prevalence and distribution of CMV genotypes in whole blood specimens from 47 transplant patients and investigate the relation with demographic and clinical features. Results: Overall, $40.4 \%$ of patients were classified as single genotype (12.8\% gB1, $23.4 \%$ gB2, 4.2\% gB3); mixed genotypes were detected in $59.6 \%$. Genotype 4 was detected only in mixed cases. In comparison to single genotypes, mixed genotypes were more frequently associated with a higher duration of DNA viremia and higher peak viral load. Conclusions: Mixed infections seem to be prevalent in Italian transplant patients; it is likely that mixed infections are more difficult to control by immunological response in comparison to single genotype infections. In this context, the genetic profile of infecting viruses and relation to clinical outcome should be investigated, also taking into account the CMV-specific cellular immune response.

๑) 2018 S. Karger AG, Basel

Cytomegalovirus (CMV) is an important cause of morbidity and mortality in transplant patients [1], with a combination of viral and host factors impacting on the development of CMV disease. Based on the polymorphisms in the UL55 gene sequence, encoding for glycoprotein B (gB), CMV has been subdivided into 4 genotypes [2]; gB has been implicated in crucial pathogenetic phases, including virus penetration into cells, cell-to-cell spread, and activation of the immune response $[3,4]$. Few and inconclusive data are available on the association between genotype, the development of CMV-associated

\section{KARGER}

(c) 2018 S. Karger AG, Basel

E-Mail karger@karger.com

www.karger.com/int
Cristina Costa

Microbiology and Virology Unit

University Hospital Città della Salute e della Scienza di Torino

Corso Bramante 88, IT-10126 Turin (Italy)

E-Mail cristina.costa@ unito.it 


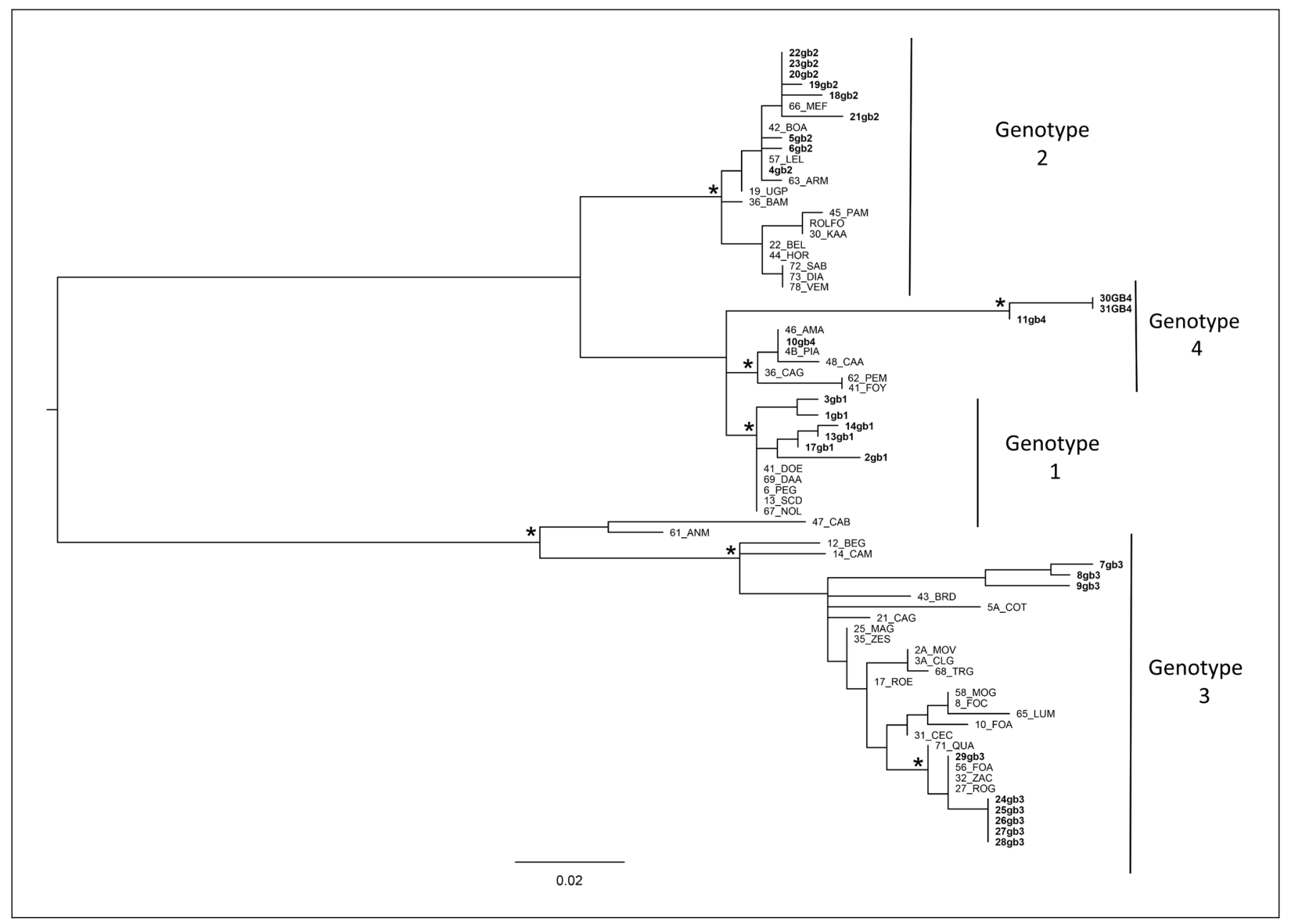

Fig. 1. Maximum likelihood phylogenetic tree of $47 \mathrm{CMV}$ gB (UL55) gene sequences plus 28 CMV genotype reference sequences. Branch lengths were estimated with the best-fitting nucleotide substitution model $(\mathrm{HKY}+\mathrm{I}+\mathrm{G})$ according to a hierarchical likeli-

disease, and clinical outcomes of transplant patients, and there are no data on $\mathrm{gB}$ prevalence and distribution in Italian case records $[5,6]$.

Herein, the prevalence and distribution of CMV gB genotypes in a group of Italian transplant patients, including hematopoietic stem cell (HSCT) and solid organ transplant (SOT) recipients, were studied by phylogenetic analysis; the relation between CMV genotype, demographic and clinical features was investigated.

Over a 1-month period, all consecutive transplant patients referred to our laboratory for posttransplant virological monitoring who tested positive for CMV-DNA on whole blood were included in the study. In detail, there were 24 SOT patients ( 9 kidney, 8 liver, 2 liver and kidney, hood ratio test, and were drawn to scale with the bar at the bottom indicating 0.09 nucleotide substitutions per site. An asterisk $\left(^{*}\right)$ along a branch represents significant statistical support for the clade subtending that branch (bootstrap support $>70 \%$ ).
4 lung, 1 heart) and 23 HSCT recipients, accounting for a total of 47 patients (male/female, 26/21; mean age $\pm S D$, $50.4 \pm 22.7$ years, median, 50 , range $3-79$ ). Informed written consent was obtained from all patients; the study was conducted in accordance with ethical standards and Helsinki Declaration, and approved by the institutional review board. CMV-DNA quantitation was determined by a commercially available real-time PCR assay (CMV ELITe MGB ${ }^{\circledR}$, ELITech Italy, Trezzano sul Naviglio, Italy) on an ABI 7500 Fast Dx Real-Time PCR platform (Applied Biosystems, Foster City, CA, USA), following automated extraction with a Qiasymphony instrument (Qiagen, Hilden, Germany). According to local practices, patients are monitored for CMV twice per week in the 
Fig. 2. Likelihood mapping analysis. Each dot represents the likelihood of the 3 possible unrooted trees for a set of 4 sequences (quartets) selected randomly from the data set. Dots close to the corners or the sides represent a tree-like phylogenetic signal (fully resolved phylogenies in which 1 tree is clearly better than the others) or network-like phylogenetic signal in the data (3 regions in which it is not possible to decide between 2 topologies), respectively. The central area represents a star-like signal (i.e., the region in which the star tree is optimal). When more than $30 \%$ of the dots fall into the center of the triangle, the data are considered unreliable for the purposes of phylogenetic inference. The percentage of dots falling in the central area of the triangle was $6.8 \%$ : the dataset showed no more than $30 \%$ noise, therefore it contained a sufficient phylogenetic signal.

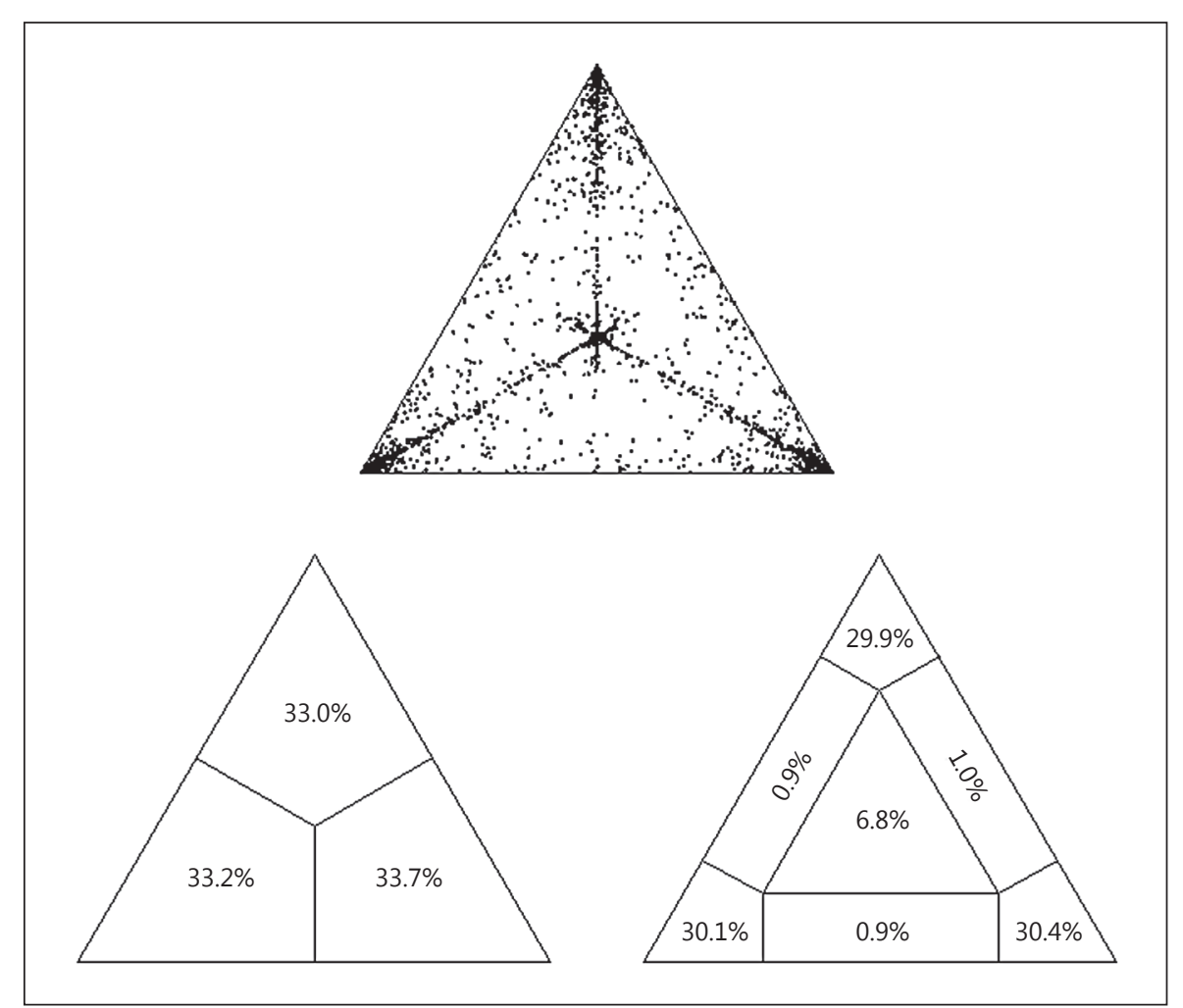

first month posttransplantation, then every 14 days until 3 months, and subsequently at 3-month intervals or on clinical indication. In the presence of CMV-DNA positivity, patients were further evaluated twice per week until negativity. Therefore, for each patient included in the study, over the subsequent 3-month period, between 1 and 6 whole blood specimens were collected, accounting for a total number of 93 specimens.

A dataset including 47 CMV UL55 gene sequences from as many study patients plus 28 genotype-specific reference sequences obtained from the National Centre for Biotechnology Information (NCBI) was built and used to establish the genotype. The phylogenetic signal generated by the dataset was investigated by the likelihood mapping analysis of 10,000 random quartets generated using TreePuzzle, as already described [7]. The sequences of the dataset were aligned using Clustal $\mathrm{X}$ and manually edited by Bioedit, as previously described [8]. The evolutionary model was chosen for the dataset as the best-fitting nucleotide substitution model in accordance with the results of the HLRT (hierarchical likelihood ratio test) implemented in Modeltest software version 3.7 [9]. The genotype of the isolates was determined by phylogenetic analysis; the maximum likelihood phylogenetic tree
Table 1. Estimates of evolutionary divergence over sequence pairs between (intra-genotypes) and within (inter-genotypes) groups

\begin{tabular}{lllll}
\hline & \multicolumn{4}{l}{ Genotype } \\
\cline { 2 - 5 } & 1 & 2 & 3 & 4 \\
\hline Genotype 1 & 0.005 & & & \\
Genotype 2 & 0.049 & 0.01 & & \\
Genotype 3 & 0.152 & 0.146 & 0.021 & \\
Genotype 4 & 0.016 & 0.053 & 0.142 & 0.014 \\
\hline
\end{tabular}

Overall, genotype 3 is more heterogeneous, as is also shown in the intra-genotype divergence distance. $p<0.005$ for all genetic distances.

was generated with the $\mathrm{HKY}+\mathrm{I}+\mathrm{G}$ model of nucleotide substitution, using Phyml v.3.0 [10].

The statistical robustness and reliability of the branching order within the phylogenetic trees was confirmed by the bootstrap analysis, considering as significant statistical support a bootstrap value $>70 \%$. The evolutionary distances among different genotypes were calculated with MEGA6 [10]. Both intra- and inter-genotype distance analyses were performed. 
Table 2. Patient features according to gB genotype

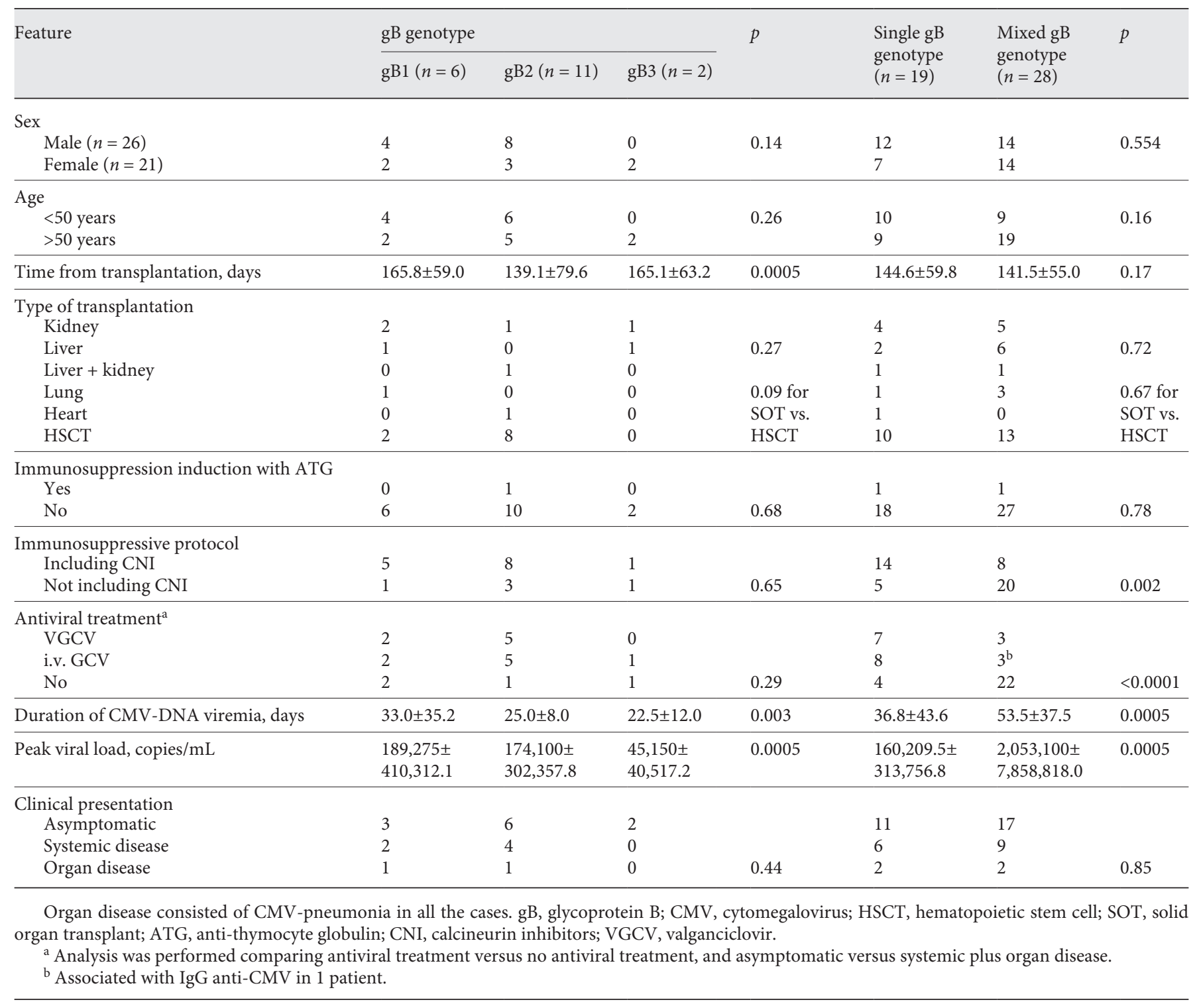

For statistical analysis, the $\chi^{2}$ test, $t$ test, and ANOVA were used, as appropriate. A $p$ value $<0.05$ was considered statistically significant.

The maximum likelihood tree (Fig. 1) of the dataset identified different statistically supported clusters, in agreement with the genotype split. The likelihood mapping analysis is presented in Figure 2 and the genetic distance in Table 1.

Considering CMV gB (UL55) gene isolates, 54/83 (65.1\%) specimens from 19 (40.4\%) patients were classified as a single gB genotype: 6 patients $(12.8 \%)$ as gB1, 11 (23.4\%) as gB2, and $2(4.2 \%)$ as gB3. Mixed gB genotypes were detected In the remaining 28 patients (59.6\%). Genotype 4 was detected only in mixed cases $(n=7)$.

Patient characteristics according to gB genotypes are provided in Table 2 . Statistically significant associations were evidenced between mixed $\mathrm{gB}$ genotypes, in comparison to single genotypes, and immunosuppressive protocols not including calcineurin inhibitors, higher duration of DNA viremia, and higher peak viral load. It is of note that the administration of antiviral therapy occurred less frequently in patients with mixed genotypes. Considering single genotypes, a higher duration of viremia was found for $\mathrm{gB} 1$, and $\mathrm{gB} 3$ was associated with a lower peak viral 
load. No significant role of gB genotypes was found for other demographic and clinical features.

Mixed infections were more frequent than infection from a single genotype (59.6 vs. $40.4 \%)$. This was not significantly different from the VICTOR study by Manuel et al. [6] on 239 SOT recipients with CMV disease in which $49 \%$ of patients displayed mixed infections. Other studies by the same research group reported a lower prevalence of mixed infections; however, these populations represented selected and smaller subgroups from patients of the VICTOR study and/or specimens other than whole blood were also included in the evaluation. The tendency toward a higher prevalence in our population in comparison to that described by Manuel et al. [6] (59.6 vs. $49 \%$ ) could be due to the higher frequency of CMV serostatus donor/recipient $\mathrm{D}+/ \mathrm{R}+$, as our population only included seropositive recipients and only 2 of them were from seronegative donors ( 1 with a gB2 genotype and 1 with a mixed genotype). Also in the study by Manuel et al. [6], mixed infection was more likely if both the donor and recipient were seropositive. Considering single gB genotypes, $12.8,23.4$, and $4.2 \%$ of patients displayed a $\mathrm{gB} 1, \mathrm{gB} 2$, and $\mathrm{gB} 3$ genotype, respectively, whereas gB4 was never found as a single infecting virus. In contrast to the data from other studies $[11,12]$ in which $\mathrm{gB} 1$ was more frequent, particularly in immunosuppressed subjects, we found a higher rate of $\mathrm{gB} 2$; however, the limitations due to the small number of individuals in each group must be considered. Interestingly, gB4 was the least represented genotype in several studies, including ours, in which it only occurred in mixed infections.

Regarding the relation between demographic and clinical features, a limitation for comparison between single genotypes is represented by the small number for each gB type, whereas comparison between single versus mixed infections could be more carefully evaluated. A significant association between gB2 and a lower time to CMV reactivation from transplantation was found, with infection occurring almost 1 month before other single gB genotypes. However, no difference was found between single $\mathrm{gB}$ genotypes versus mixed $\mathrm{gB}$ infections. On the other hand, the duration of viremia was significantly higher in $\mathrm{gB} 1$ in comparison to other single genotypes. A highly significant association was found between mixed infections and an increased duration of CMV-DNA positivity, as well as peak viral load. Different determinants are likely to contribute to these findings. First, antiviral treatment was not administered in most of the patients with mixed infections (or at least at first CMV positivity detection for patients with serial specimens), thus allowing for a longer viremia. Second, the failure of antiviral therapy is more likely in mixed infections, as previously reported $[6,13]$. Previous studies [6] have evidenced that mixed gB infections were a significant predictor of failure to eradicate CMV at 3 weeks. In the present study, the mean duration of viremia was significantly higher for mixed versus single genotype infections. It could be hypothesized that mixed infections are more difficult to control through an immunological response. In this context, as CMV replication is mainly under the control of the T-cell immune response, evaluation of CMV-specific cellular immune responses, for example by Elispot assay, could be of interest. Unfortunately, at the time of specimen collection at our center, Elispot assay was routinely performed only in kidney transplant recipients [14]. Therefore, data were available only in a minority of subjects (5 out of 9 kidney transplant recipients, and 2 kidney and liver transplant recipients). Among these individuals, 2 presented a single $\mathrm{gB}$ infection and 3 a mixed infection; 1 from each group displayed a CMV responder status by Elispot assay, therefore no analysis could be performed. Further studies evaluating the relation between clinical outcome and $\mathrm{gB}$ genotypes should be performed, taking this into consideration and also that it could be interesting to evaluate patterns of cellular immune response using a peptide mix containing $\mathrm{gB}$ as the antigenic stimulus. As discussed in the study by Manuel et al. [6], seropositive recipients are likely to display a preexisting cellular immune response only to antigenic epitopes of their own viral strains, but not to the donor strains. Therefore, seropositive recipients could act as nonresponders/weak responders to the newly acquired CMV types.

In conclusion, by using phylogenetic analysis in a group of Italian SOT and HSCT patients, a high prevalence of mixed $\mathrm{gB}$ infections and a significant association between these and an increased level of viral replication, in terms of duration of viremia and peak viral load, were found. A possible role of cellular immune control in this context could be hypothesized and should be investigated specifically.

\section{Disclosure Statement}

The authors have no conflicts of interest to disclose. 


\section{References}

1 Ciotti M, Kessler HH: Pathogens relevant in transplantation medicine; in Kessler $\mathrm{HH}$ (eds): Molecular Diagnostics of Infectious Diseases. Berlin, De Gruyter, 2014, p 105.

2 Chou SW, Dennison KM: Analysis of interstrain variation in cytomegalovirus glycoprotein $\mathrm{B}$ sequences encoding neutralization related epitopes. J Infect Dis 1991;163:12291234.

3 Navarro D, Paz P, Tugizov S, Topp K, La Vail J, Pereira L: Glycoprotein B of human cytomegalovirus promotes virion penetration into cells, transmission of infection from cell to cell, and fusion of infected cells. Virology 1993;197:143-158.

4 Boehme KW, Singh J, Perry ST, Compton T: Human cytomegalovirus elicits a coordinated cellular antiviral response via envelope glycoprotein. B J Virol 2004;78:1202-1211.

5 Dieamant DC, Bonon SHA, Peres RMB, Costa CRC, Albuquerque DM, Miranda ECM, Aranha FJP, Oliveira-Duarte G, Fernandes VCA, De Souza CA, Costa SCB, Vigorito AC: Cytomegalovirus (CMV) genotype in allogeneic hematopoietic stem cell transplantation. BMC Infect Dis 2013;13:310.
6 Manuel O, Asberg A, Pang X, Rollag H, Emery VC, Preiksaitis JK, Kumar D, Pescovitz MD, Bignamini AA, Hartmann A, Jardine AG, Humar A: Impact of genetic polymorphisms in cytomegalovirus glycoprotein b on outcomes in solid-organ transplant recipients with cytomegalovirus disease. Clin Infect Dis 2009;49:1160-1166.

7 Strimmer K, von Haeseler A: Likelihoodmapping: a simple method to visualize phylogenetic content of a sequence alignment. Proc Natl Acad Sci USA 1997;94:6815-6819.

8 Ciccozzi M, Lo Presti A, Cella E, Giovanetti M, Lai A, El-Sawaf G, Faggioni G, Vescio F, Al Ameri R, De Santis R, Helaly G, Pomponi A, Metwally D, Fantini M, Qadi H, Zehender G, Lista F, Rezza G: Phylogeny of Dengue and Chikungunya viruses in Al Hudayda governorate, Yemen. Infect Genet Evol 2014;27: 395-401.

9 Posada D, Buckley TR: Model selection and model averaging in phylogenetics: advantages of Akaike Information Criterion and Bayesian approaches over likelihood ratio tests. Syst Biol 2004;53:793-808.
10 Tamura K, Stecher G, Peterson D, Filipski A, Kumar S: MEGA6: Molecular Evolutionary Genetics Analysis version 6.0. Mol Biol Evol 2013;30:2725-2729.

11 Wu KG, Hung MC, Chang YT, Chen CJ, Yang SP, Liu CY, Ho DM, Chan YJ: Occurrence of human cytomegalovirus glycoprotein B genotypes in immunocompetent and immunosuppressed Taiwanese patients. Intervirology 2011;54:196-201.

12 Xia CS, Zhao XT, Sun YY, Zhang Z: Human cytomegalovirus glycoprotein $B$ genotypes in Chinese hematopoietic stem cell transplant recipients. Intervirology 2012;55:342-348.

13 Coaquette A, Bourgeois A, Dirand C, Varin A, Chen W, Herbein G: Mixed cytomegalovirus glycoprotein B genotypes in immunocompromised patients. Clin Infect Dis 2004; 39:155-161.

14 Costa C, Balloco C, Sidoti F, Mantovani S, Rittà $\mathrm{M}$, Piceghello $\mathrm{A}$, Fop F, Messina M, Cavallo R: Evaluation of CMV-specific cellular immune response by EliSPOT assay in kidney transplant patients. J Clin Virol 2014;61:523528. 